\title{
Impactación Canina Maxilar y Reabsorción Radicular de Dientes Adyacentes: Un Análisis a Través de Tomografía Computarizada Cone-Beam
}

\author{
Canine Maxillary Impaction and Root Resorption of Adjacent Teeth: \\ An Analysis through Cone-Beam Computed Tomography Analysis
}

Miguel Muñoz Domon'; Daniel Arraya Valdés²; Daniela Castro Catalán ${ }^{3}$ \& Cristian Vergara Núñez ${ }^{4}$

MUÑOZ, D. M.; ARRAYA, V. D.; CASTRO, C. D. \& VERGARA, N. C. Impactación canina maxilar y reabsorción radicular de dientes adyacentes: un análisis a través de tomografía computarizada cone-beam. Int. J. Odontostomat., 14(1):27-34, 2020.

RESUMEN: Uno de los problemas con mayores consecuencias tanto estéticas como funcionales en ortodoncia es la impactación de caninos maxilares (ICM) siendo principal motivo de reabsorciones radiculares de dientes adyacentes. Actualmente se carece de estudios que describan la reabsorción radicular por ICM a través de tomografía computarizada conebeam (TCCB) en la población chilena. El objetivo del estudio fue describir a pacientes entre 8 a 18 años, que presentan ICM (o riesgo de ella), tanto en las características de la impactación canina como en la reabsorción radicular presentada en incisivos laterales (IL) y en otros dientes adyacentes. En este estudio retrospectivo, 135 datos de TCCB fueron seleccionados por conveniencia, de los cuales 181 caninos presentaron ICM. Fueron analizadas las variables edad, sexo, tipo de ICM (unilateral o bilateral), localización bucolingual (vestibular o palatino), lado de impactación (derecho o izquierdo), reabsorción radicular en el incisivo lateral y registro de el o los dientes anexos afectados por reabsorción radicular (incisivo central y/o premolares). Además en el caso de reabsorción de IL fue analizada la reabsorción tridimensionalmente a través de la ThreeDimensional Leeds Orthodontic Root Resorption Target Scale (3D-LORTS). La ICM en la muestra seleccionada tuvo una edad promedio de 12 años, una relación por sexo mujeres:hombres de 1,5:1, frecuentemente de tipo unilateral, en el lado derecho y una localización bucolingual palatina $(64,1 \%)$ por sobre la vestibular (35,9 \%). La reabsorción radicular alcanzó un 35,9 \%, de la cual un $27,6 \%$ involucró a IL y un $8,3 \%$ en dientes anexos adyacentes. En cuanto a la distribución tridimensional de la reabsorción radicular, la más frecuente involucró el tercio apical (60\%), con compromiso pulpar o severo (40\%), involucrando solo una cara, la palatina (54\%). Ninguna de las variables anteriores consideradas, demostró diferencias estadísticas con la reabsorción radicular de incisivos laterales.

PALABRAS CLAVE: impactación canina, diente, cone beam, reabsorción radicular.

\section{INTRODUCCIÓN}

Uno de los problemas con mayores consecuencias tanto estéticas como funcionales en ortodoncia es la impactación de caninos maxilares (ICM). Éstos presentan el más largo periodo de descenso desde su lugar de formación (lateral a la fosa piriforme) hasta su erupción en la arcada (Dewel, 1949), esta situación favorece a que sean susceptibles a alteraciones de su trayecto y su impactación, siendo esta última uno de los principales motivos de reabsorciones radiculares de dientes adyacentes. Las causas de la ICM se pueden clasificar en cuatro grupos distintos, según Becker \& Chaushu (2015): obstrucción local del tejido duro, patología local, desviación o alteración del desarrollo normal de los incisivos y factores hereditarios o genéticos. Dos teorías destacan como explicación de la ICM: la teoría genética y la guía de erupción del canino (Jacoby, 1983; Yan et al., 2013).

\footnotetext{
${ }^{1}$ Universidad de Chile, Centro de Referencia Odontopediátrico Simón Bolívar, Servicio de Salud Viña del Mar-Quillota, Viña del Mar, Chile.

2 Facultad de Odontología, Universidad Andrés Bello, Viña del Mar, Chile.

${ }^{3}$ Universidad Andrés Bello, Viña del Mar, Chile.

${ }^{4}$ Universidad de Chile, Profesor Asistente, Departamento del Niño y Ortopedia Dentomaxilar, Universidad de Chile, Santiago, Chile.
} 
La prevalencia en la población se delimita entre el 1 al $3 \%$ (Alqerban et al., 2009), recientes estudios en la población mexicana hablan de un 6,04 \% (HerreraAtoche et al., 2017), mientras que la población chilena bordea el 2,3\% (Bizcar et al., 2015). Se ha reportado que la frecuencia de ICM palatina es el doble que la vestibular en población caucásica, no así la asiática donde la más frecuente es la vestibular (Alqerban et al.). En cuanto a sexo, las mujeres presentan mayor frecuencia que los hombres, algunos autores hablan de hasta tres veces más (Sacerdoti \& Baccetti, 2004) sin embargo, no siempre se encuentra una distribución estadísticamente significativa, pero si se ha demostrado la mayor frecuencia en mujeres (Becker \& Chaushu).

Se describen como métodos de evaluación de ICM, el examen visual, palpación clínica y métodos imagenológicos; de este último clásicos son la ortopantomografía y la tomografía computarizada conebeam (TCCB). La precisión diagnóstica y el aporte a la planificación previa al tratamiento, sin la sobreposición de estructuras en los tres sentidos del espacio, hace hoy en día del TCCB por sobre el examen radiográfico, el exámen complementario ideal en caso de ICM (Alqerban et al.); esto demostrado y ejemplificado a través del pionero estudio de reabsorciones radiculares por ICM de Ericson \& kurol (2000) donde, por medio de tomografía computarizada (TC), la frecuencia de reabsorciones radiculares reportada ascendió a un 38 $\%$ en incisivos laterales al diagnosticar por TC, distante del $12.5 \%$ encontrado por ellos mediante examen radiográfico (Ericson \& Kurol,1987).

Actualmente se carece de estudios que describan la reabsorción radicular por ICM a través de TCCB en la población chilena, encontrándose solamente estudios de prevalencia realizados a través de ortopantomografía, motivo por el cual es que el presente estudio propuso describir a pacientes entre 8 a 18 años que presentan ICM (o riesgo de presentarla), tanto en las características propias de la impactación canina, como en la reabsorción radicular presentada en incisivos laterales y en otros dientes adyacentes a la impactación.

\section{MATERIAL Y MÉTODO}

En esta investigación de carácter retrospectivo, 135 datos de TCCB ligados a sexo y edad, fueron obtenidos de la base de datos imagenológica del Centro de Referencia Odontopediátrico Simón Bolívar
(CROSB), Servicio de Salud Viña del Mar-Quillota (SSVQ), Viña del Mar-Chile. Los TCCB pertenecen a pacientes referidos al centro desde otros servicios de salud públicos de la red del SSVQ o bien por consulta en dicho centro hasta noviembre de 2018. 89 de los datos de TCCB presentaron ICM unilateral, mientras que 46 fueron bilaterales, por tanto, un total de 181 caninos en riesgo de impactación o impactación canina fueron evaluados. Todos los datos pertenecen a pacientes con el diagnóstico de ICM o en riesgo de impactación, establecido clínica y radiográficamente con ortopantomografía. Los criterios de inclusión de la muestra fueron TCCB de pacientes: (1) entre 8 años y 18 años en el momento de la captura de los datos del TCCB con diagnóstico clínico-radiográfico (a través de ortopantomografía) de ICM o en riesgo de impactación. Los criterios de exclusión fueron pacientes: (1) con tratamiento ortodóncico anterior, (2) historial en la base de datos de trauma dentoalveolar o cirugía maxilar anterior (3) fisura labiopalatina o sus variantes (4) síndrome de expresión maxilofacial documentada en la base de datos y (5) antecedente de onicofagia reportado en la base de datos. Todos los TCCB fueron indicados por sus respectivos ortodoncistas tratantes ya sea por razones de ahondar en un diagnóstico más específico, sospecha de reabsorción o visión necesaria por el tratante por razones de tratamiento.

Los registros de TCCB se obtuvieron con los pacientes en posición vertical utilizando el mismo equipo de TCCB (Planmeca Promax 3D Classic, Illinois, USA) y los mismos parámetros: campo de visión de $\varnothing$ 80 × $80 \mathrm{~mm}, 60-90 \mathrm{kV}, 1-14 \mathrm{~mA}$ y tamaño de vóxel de $200 \mu \mathrm{m}$. Los datos de los TCCB fueron guardados en archivos en formato Digital Imaging and Communication in Medicine (DICOM) los cuales les fueron asignadas nuevas identificaciones utilizando códigos generados aleatoriamente que eliminaron la información de identificación original del paciente. Los datos fueron exportados al software Planmeca Romexis ${ }^{\circledR}$ Viewer (Planmeca OY, Helsinki, Finlandia). Posteriormente las imágenes de los TCCB fueron analizadas por un solo investigador M.M.D. Especialista en Ortodoncia y Ortopedia Dento Máxilo Facial y registrados en una base de datos planilla MS Excel 2019 por el investigador D.A.V.. Las imágenes fueron analizadas en el mismo lugar (CROSB), computador (HP compaq 8200 elite small form factor), monitor (Samsung LED S22B300, resolución $1920 \times 180$, frecuencia $60 \mathrm{~Hz}$ y colores a 32 bits) y a la misma hora del día entre las 07:00 y 08:30 horas por un periodo de 2 semanas. Las evaluaciones fueron hechas en vistas de cortes axiales, sagitales y 
coronales de reconstrucción multiplanar (RMP) y en reconstrucción tridimensional (3D) de superficie sombreada en vistas frontal y lateral derecha e izquierda ambas no estrictas. Los cortes son mostrados en las Figura 1 y 2

En el análisis, fueron evaluadas las variables: edad, sexo, tipo de ICM (unilateral o bilateral), localización bucolingual (vestibular o palatino), lado de impactación (derecho o izquierdo), reabsorción radicular en el incisivo lateral (IL) (su presencia o no), registro del o los dientes anexos o adicionales afectados por reabsorción radicular (incisivo central, primer premolar y/o segundo premolar). Además, en aquellos casos donde se presentó reabsorción radicular en el IL fue utilizada la escala de evaluación propuesta por Jaward et al. (2016), Three-Dimensional Leeds Orthodontic Root
Resorption Target Scale o 3D-LORTS. Esta escala evalúa en forma de variables categóricas la reabsorción, las cuales están descritas en la Tabla I. Respecto a la variable profundidad en la escala 3D-LORTS, el valor "Medio", fue modificando de la forma original (Dentina) planteada por Jaward et al., considerando la reabsorción independiente si considera menos o más del $50 \%$ de la dentina radicular, esto a razón de la dificultad de calibración. Las categorías de esta variable son equivalentes a la reabsorción leve, moderada o severa planteadas por Ericson \& Kurol (2000). Los datos recolectados en la base de datos fueron posteriormente analizados en el software R Projet v.3.5.3 (R Foundation for Statistical Computing, Vienna, Austria) donde fue aplicada estadística descriptiva e inferencial, en esta última fueron aplicados test de chi-cuadrado para determinar relación entre variables. Se consideraron significativos valores de $p \leq 0,05$.

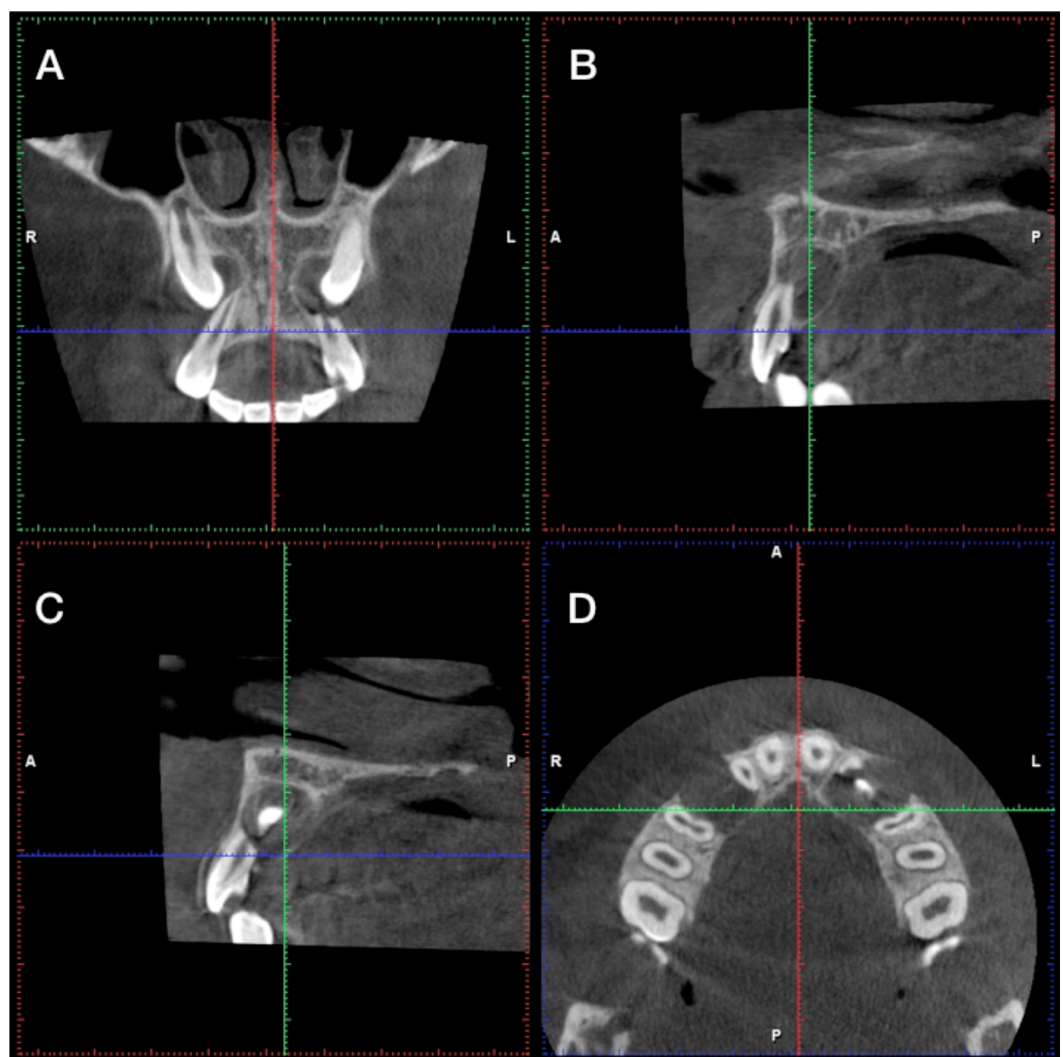

Fig. 1. Cortes de reconstrucción multiplanar de TCCB. Paciente sexo masculino, 12 años de edad. A) corte coronal, B) corte sagital en relación a eje de diente $1.2, \mathrm{C}$ ) corte sagital en relación a eje de diente 2.2 , D) corte axial. La combinación de las cuatro observaciones sumado a la reconstrucción 3D en Fig. 2 determina ICM bilateral, en posición palatina, reabsorción radicular en dientes 1.2 y 2.2; con compromiso del tercio apical, profundidad pulpar e involucrando todas sus caras en diente 1.2 y de tercio apical-medio, profundidad pulpar e involucrando todas sus caras en diente 2.2 (adicionalmente los caninos presentaron diagnóstico de capuchón pericoronario compatible con quiste dentígero).
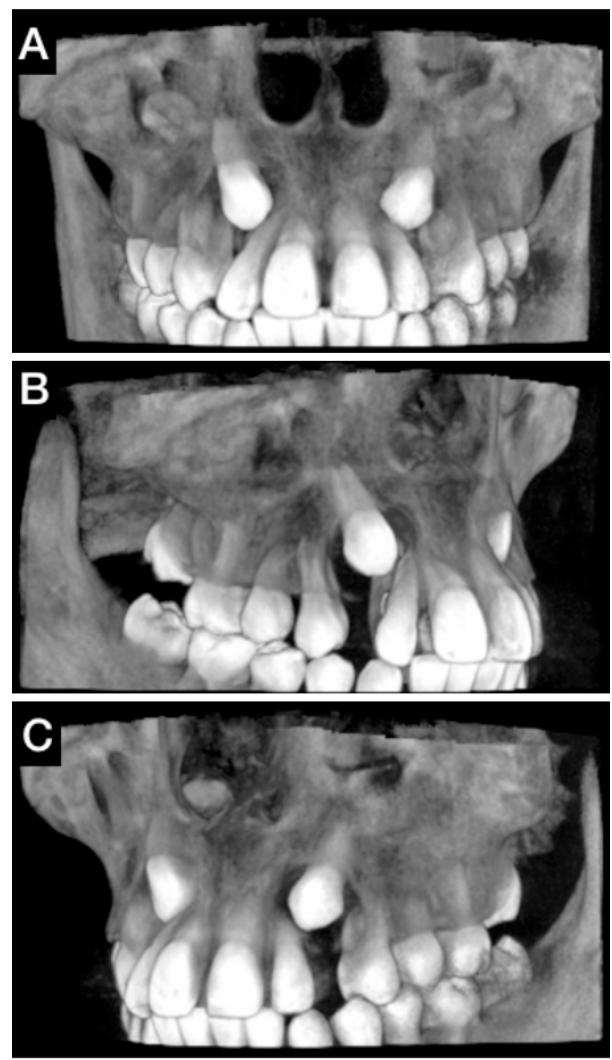

Fig. 2. Reconstrucción 3D de superficie sombreada. Mismo paciente descrito en Figura 1. A) vista frontal, B) vista lateral no estricta derecha, posición palatina de diente 1.3 con capuchón pericoronario adyacente a reabsorción radicular de diente $1.2, \mathrm{C}$ ) vista lateral no estricta izquierda, posición palatina de diente 2.3 con capuchón pericoronario en relación a reabsorción radicular de diente 2.2 . 
MUÑOZ, D. M.; ARRAYA, V. D.; CASTRO, C. D. \& VERGARA, N. C. Impactación canina maxilar y reabsorción radicular de dientes adyacentes: un análisis a través de tomografía computarizada cone-beam. Int. J. Odontostomat., 14(1):27-34, 2020

Tabla I. Criterios de evaluación del compromiso tridimensional de reabsorción radicular de incisivos laterales producto de ICM a través de 3D-LORTS.

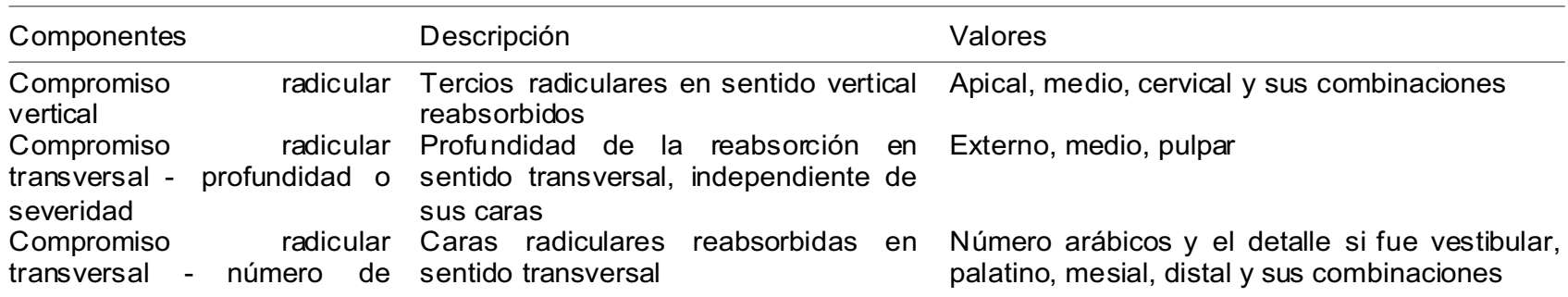

\section{RESULTADOS}

De los 135 sujetos, entre 8 - 18 años, la edad promedio fue de $12 \pm 2,26$ años, donde la mayor concentración de datos (50\%) se encontró entre los 10 a 13 años. La distribución por sexo resultó de un 60,7 \% mujeres y $39,3 \%$ hombres. Respecto a las mujeres, estas presentaron mayor concentración de datos entre los 11 y 13 años, mientras que en los hombres se mantuvieron medidas homogéneas en su distribución, lo anterior es mostrado en la Figura 3. En cuanto al tipo de ICM, se obtuvo que $65,9 \%$ presentaron impactación unilateral y $34,1 \%$ bilateral. Respecto a la localización bucolingual de los ICM 64,1 \% presentaron localización palatina, 35,9 \% vestibular; 54,1\% se ubicaron en el lado derecho y $45,9 \%$ en el lado

Tabla II. Distribución de reabsorción radicular según diente afectado.

\begin{tabular}{lll}
\hline Diente afectado & \multicolumn{2}{c}{ Reabsorción } \\
& Frecuencia & Porcentaje \\
\hline Incisivo lateral & 50 & $76,9 \%$ \\
Incisivo central & 8 & $12,3 \%$ \\
1er Premolar & 6 & $9,2 \%$ \\
2do Premolar & 1 & $1,5 \%$
\end{tabular}

izquierdo. La reabsorción radicular fue de un 35,9 \% involucrando incisivos laterales y/o un diente anexo. La reabsorción radicular que afectó a incisivos laterales (IL) fue de un $27,6 \%$, mientras que $72,4 \%$ no presentaron esa condición. De los IL que presentaron reabsorción, el 18,2 \% corresponde a mujeres mientras que el 9,4 \% a hombres. Por otra parte, 8,3\% afectaron a un diente anexo (incisivo central o premolar), de los cuales seis casos afectaron el lado derecho y nueve el lado izquierdo. El detalle de la distribución de la reabsorción radicular por diente afectado se muestra en la Tabla II. En cuanto a la distribución y características del compromiso tridimensional en aquellos casos donde fue afectado el IL por reabsorción radicular es expuesto en la Tabla III. Es importante señalar además que en cuanto al compromiso transversal (número de caras), cuando la reabsorción involucró a una cara, la más afectada fue la palatina (11 casos), cuando fueron dos caras la disto-palatina (8 casos) y cuando involucró tres caras la disto-palato-vestibular (11 casos). Finalmente, no se encontraron relaciones entre las variables estudiadas:

Tabla III. Distribución del compromiso tridimensional de reabsorción radicular de incisivos laterales producto de ICM a través de 3D-LORTS.

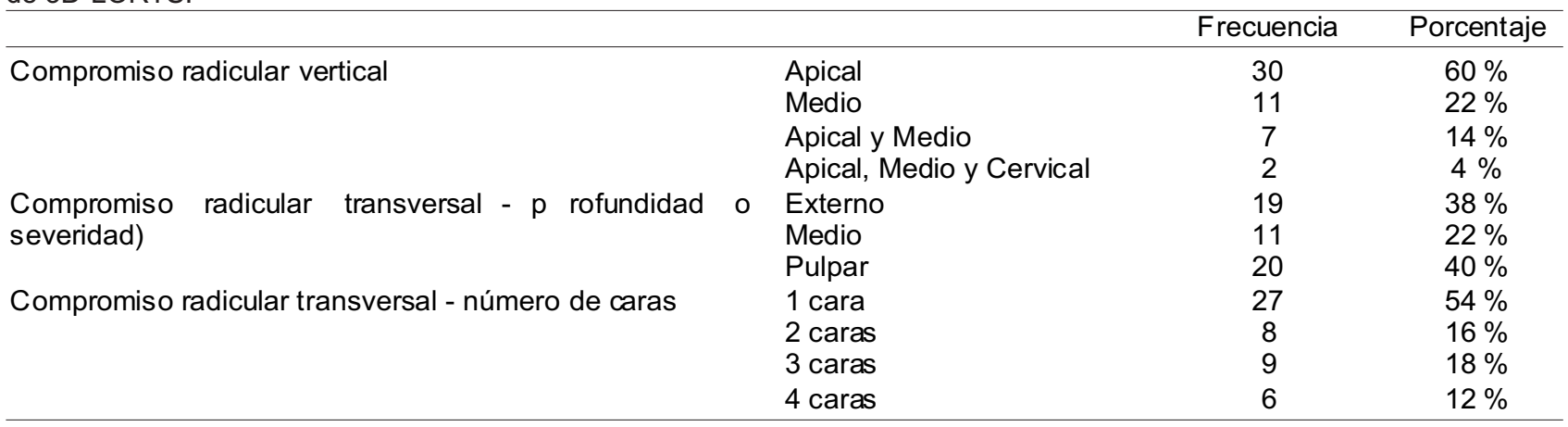


MUÑOZ, D. M.; ARRAYA, V. D.; CASTRO, C. D. \& VERGARA, N. C. Impactación canina maxilar y reabsorción radicular de dientes adyacentes: un análisis a través de tomografía computarizada cone-beam. Int. J. Odontostomat., 14(1):27-34, 2020.

edad, sexo, tipo de impactación, lado de impactación y posición bucolingual con la reabsorción radicular de
IL producto de impactación canina, tal como muestra la Tabla IV.

Tabla IV. Relación entre la edad, sexo, tipo de impactación, lado de impactación y posición bucolingual con la reabsorción radicular de incisivos laterales producto de impactación canina.

\begin{tabular}{|c|c|c|c|c|}
\hline \multirow{2}{*}{$\begin{array}{l}\text { Variables } \\
\text { Edad }\end{array}$} & 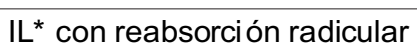 & $\mathrm{IL}^{*} \sin$ reabsorción radicular & $x^{2}$ & Valor de $p^{* *}$ \\
\hline & & & 1,9 & 0,76 \\
\hline 8 - 9 años & $5,9 \%(8)$ & $8,1 \%(11)$ & & \\
\hline $10-11$ años & $8,1 \%(11)$ & $19,3 \%(26)$ & & \\
\hline $12-13$ años & $13,3 \%(18)$ & $23 \%(31)$ & & \\
\hline $14-15$ años & $3,7 \%(5)$ & $11,9 \%(16)$ & & \\
\hline $16-18$ años & $3 \%(4)$ & $3,7 \%(5)$ & & \\
\hline Sexo & & & 0,99 & 0,32 \\
\hline Hombres & $11,9 \%(16)$ & $27,4 \%(3,7)$ & & \\
\hline Mujeres & $22,2 \%(30)$ & $38,5 \%(52)$ & & \\
\hline Tipo de Impactación & & & 0,94 & 0,33 \\
\hline Unilateral & $20,7 \%(28)$ & $45,2 \%(61)$ & & \\
\hline Bilateral & $13,3 \%(18)$ & $20,7 \%(28)$ & & \\
\hline Lado de Impactación & & & 1,42 & 0,23 \\
\hline Derecha & $36,5 \%(66)$ & $17,7 \%(32)$ & & \\
\hline Izquierda & $17,7 \%(32)$ & $28,2 \%(51)$ & & \\
\hline Posición Bucolingual & & & 0,25 & 0,61 \\
\hline Palatino & $22,7 \%(41)$ & $41,4 \%(75)$ & & \\
\hline Vestibular & $12,7 \%(23)$ & $23,2 \%(42)$ & & \\
\hline
\end{tabular}

$\left.{ }^{*}\right) \mathrm{IL}=$ Incisivo lateral. ${ }^{(*)}$ Las hipótesis fueron contrastadas con un nivel de significancia $p=0.05$, equivalente a asumir un $5 \%$ del error tipo I.

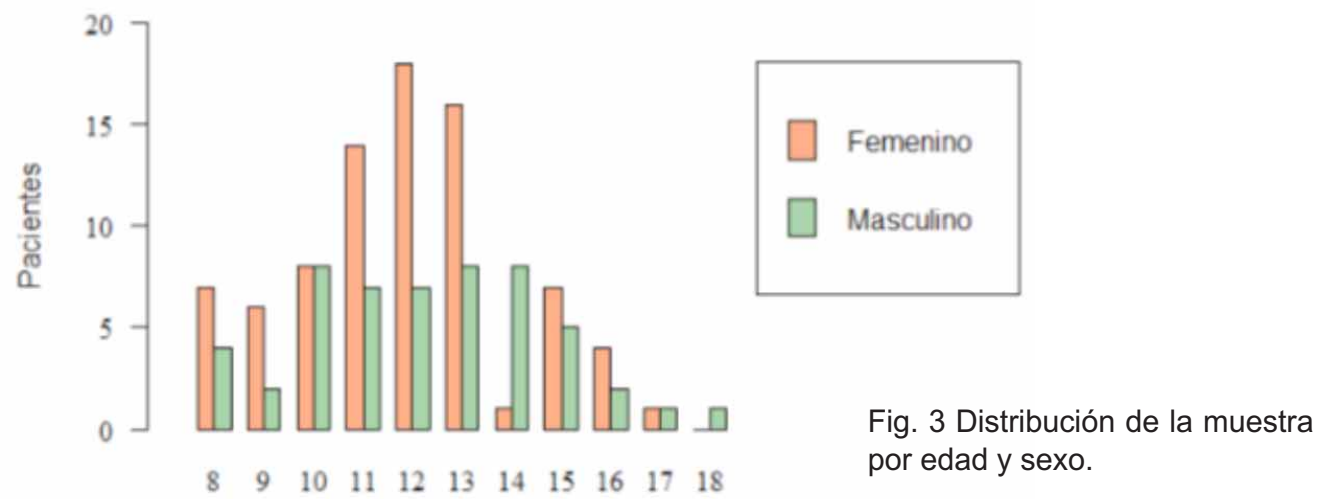

Edad (años)

\section{DISCUSIÓN}

Respecto a las características de la muestra seleccionada, fue encontrada una predominancia de mujeres por sobre hombres en el grupo de estudio, siendo el $60,7 \%$ mujeres (M) y el 39,3\% hombres $(H)$, con una relación de 1,5:1 respectivamente, este dato es congruente a lo encontrado previamente en la población chilena, con una relación 1,6:1 (Bizcar et al.). Si bien existe variabilidad en la literatura respecto a esta relación M:H, como la relación 3:1 encontrada por Sacerdoti \& Baccetti, o de 2:1 por Lai et al. (2013) con una muestra muy similar a la de este estudio, los estudios apuntan a que predomina la ICM en mujeres (Becker \& Chaushu). En cuanto a la edad, la mayor concentración de sujetos fue entre 10 y 13 años, con una media 
de 12 años, similar media de 12.5 años encontrada por Ericson \& Kurol (2000), con una muestra seleccionada por conveniencia con similares características. Éstos además, observaron que las reabsorciones fueron frecuentes entre los 11 y 12 años. A pesar de esto, no hay evidencia de una relación significativa entre la edad con las reabsorciones radiculares (Lai et al.) algo que tampoco fue encontrado en este estudio.

La ICM de tipo unilateral $(65,9 \%)$ fue más prevalente que la bilateral $(34,7 \%)$, en una relación de 1,8:1 misma relación encontrada por Bizcar et al., y cercana a lo encontrado por Cernochova et al. (2011) con una proporción de 2,2:1, lo que indicaría un mayor predominio de la impactación unilateral. El mayor número de casos de ICM, en este estudio, se reportó en el lado derecho $(54,1 \%)$ por sobre el lado izquierdo (45,9\%), Stewart et al. (2001), también reportó una frecuencia mayor en el lado derecho, contrario a Bizcar et al. el cual observó en el lado izquierdo una mayor frecuencia.

La localización buco lingual más frecuente fue con desplazamiento hacia palatino con un $64,1 \%$, versus un 35,9\% vestibular, es decir una relación 1,8:1. Esta característica es más compleja de contrastar puesto que hay autores que consideran una tercera posición con diferentes denominaciones tales como; en la línea del arco, media o bicortical. Ericson \& Kurol (1987), quienes describen una distribución palatina y vestibular, hablan de un $80 \%$ y $20 \%$ (2:1) respectivamente, mientras que autores que consideran una categoría intermedia como Arriola-Guillén et al. (2018), Chenochova et al. y Lai et al. describen una mayor frecuencia en desplazamiento palatino, seguido de vestibular y bicortical. Sin embargo, una excepción a lo que pareciera predominar (el desplazamiento palatino) es en población asiática, según lo reportado por Liu et al., (2008) donde predomina el desplazamiento hacia vestibular.

La reabsorción radicular encontrada, alcanzó un $35,9 \%$, valor muy cercano al 38,8 \% encontrado por Lai et al. quien también observa reabsorciones radiculares en incisivos y premolares, por otra parte, la comparativa no es idónea con otros autores, como es el caso de Erikson \& Kurol (2000) o Walker et al. (2005) donde sólo evalúan incisivos y no premolares. En cuanto a la reabsorción radicular de un $27,6 \%$ en incisivos laterales se obtuvo valores cercanos a los descritos por Lai et al. quienes encontraron un 25,37 $\%$, sin embargo, existe gran heterogeneidad en cuanto a otros autores, Erikson \& Kurol (2000) con un 38 $\%$, y Cernochova et al. con un $61 \%$ de afectación de incisivos laterales. Además, es una tendencia la menor afectación porcentual del incisivo central o premolares respecto al incisivo lateral, así como se obtuvo en este estudio (Tabla II), y que está en concordancia con los mismos autores nombrados, aunque una vez más existe gran variabilidad en término de proporciones. Por otra parte, no existe consenso en la bibliografía para caracterizar la reabsorción radicular, lo cual complejiza la comparación de resultados de esta variable con otros estudios. En la búsqueda de sopesar lo anterior, en esta investigación se aplicó lo planteado por Jawad et al., que sigue descripciones intuitivas respecto a reabsorciones radiculares. En cuanto al primer aspecto de evaluación de este método, el compromiso vertical, el más frecuente encontrado es el apical, seguido por el medio, apical-medio y apical-medio-cervical (Tabla IV), datos similares obtenidos en otros estudios de población caucásica y china (Cernochova et al.; Yan et al.) con el mismo tipo de categorías, esto concuerda con la lógica de la frecuencia de distribución en población chilena en cuanto a posicionamiento vertical que sigue la ICM, independiente si genera o no reabsorción (Bizcar et al.). Sin perjuicio de lo anterior, Erikson \& Kurol (2000) encontraron que se presenta en mayor medida en el tercio medio y luego en el tercio apical, sin embargo, el investigador agrupa en la categoría del tercio medio aquellos casos que también presentan compromiso apical-medio, lo cual explicaría la no concordancia y su mayor incidencia en este tercio, misma situación ocurre con estudios más actuales (Lai et al.). Del compromiso transversal, número de caras involucradas, no existen estudios actuales que las cuantifiquen como en este estudio, pero sí describen las caras más frecuentemente involucradas, donde Ericson \& Kurol (2000) encontraron que la cara más afectada es la disto-palatina, en nuestro estudio fue la palatina cuando involucró una cara seguido de la disto palatina cuando fueron dos, lo anterior concuerda también a lo encontrado por Dogramacı et al. (2015); una vez más no es posible realizar comparaciones concluyentes, sin embargo, la región palatina y distal parecieran ser las más afectadas. En cuanto al compromiso transversal, profundidad de la reabsorción en IL, denominado también severidad de la reabsorción por otros autores, el compromiso pulpar o severo es el más frecuente (40\%) seguido por el externo o leve (38 \%) y el medio (22\%); Erikson \& Kurol (2000) encontró un $60 \%$, $31 \%$ y $9 \%$ respectivamente, mientras que Dogramaci et al. reporta un $24,7 \%$ de compromiso pulpar, y Lai et al. encuentra también una mayor incidencia en el compromiso pulpar. Esta variabilidad se puede deber a las diferencias de espacio muestral en los estudios y casos identi- 
ficados, sin embargo, todos apuntan al parecer a que predomina el compromiso pulpar en reabsorciones radiculares por ICM.

Finalmente, en la búsqueda de algún factor relacionado, como la edad, sexo, posición bucolingual, lado de impactación y tipo de impactación con la reabsorción radicular, los hallazgos de esta investigación indican que ningún factor estudiado estaría relacionado (Tabla IV), Cernochova et al., encuentra relación solamente con el factor de posición bucolingual, resultado que no ha sido descrito en estudios posteriores, como el de Yan et al. o el de Lai et al., donde en ambos tampoco hay relación con las variables estudiadas, si bien como se ha comentado, existen mayores tendencias como es el caso de la edad, sexo, tipo de impactación y la posición bucolingual, no se ha podido establecer una relación estadística. Finalmente se deben tomar en cuenta las propias limitaciones de este y la mayoría de los estudios de este tipo, donde son muestras seleccionadas por conveniencia en una población con ciertas características propias a las que asisten a determinados centros de atención en salud, por lo cual, estos hallazgos no deben extrapolarse a la población.

\section{CONCLUSIONES}

1. La ICM en la muestra seleccionada tuvo una edad promedio de 12 años, una relación por sexo M:H de $1,5: 1$, frecuentemente de tipo unilateral, en el lado derecho y una localización bucolingual palatina $(64,1 \%)$ por sobre la vestibular (35,9\%).

2. La reabsorción radicular alcanzó un $35,9 \%$, de la cual un $27,6 \%$ involucró a incisivos laterales y un 8.3 $\%$ en dientes anexos adyacentes (incisivo central o premolares).

3. En cuanto a la distribución tridimensional de la reabsorción radicular, la más frecuente involucró el tercio apical (60\%), con compromiso pulpar o severo (40 $\%)$, involucrando una sola cara, la palatina (54\%).

4. Ninguna de las variables anteriores consideradas, demostró diferencias estadísticas con la reabsorción radicular de incisivos laterales.

AGRADECIMIENTOS. Agradecemos la ayuda de Yvette Díaz-Muñoz en la revisión de traducciones.
MUÑOZ, D. M.; ARRAYA, V. D.; CASTRO, C. D. \& VERGARA, N. C. Canine maxillary impaction and root resorption of adjacent teeth: An analysis through cone-beam computed tomography. Int. J. Odontostomat., 14(1):27-34, 2020.

ABSTRACT: One of the problems with greatest aesthetic and functional consequences in orthodontics is the Impaction of Maxillary Canines (ICM) being the main reason for root resorption of the adjacent teeth. Currently there are no studies describing root resorption by ICM through cone beam computed tomography (CBCT) in the Chilean population. The main objective of the study was to describe patients between 8 and 18 years old, who presented with ICM (or risk thereof), with the characteristics of canine impaction as well as root resorption presented in lateral incisors (IL) and in other adjacent teeth. In this retrospective study, 135 CBCT subjects were selected for convenience, of which 181 canines presented ICM. The variables were analyzed: age, sex, type of ICM (unilateral or bilateral), buccolingual location (vestibular or palatal), side of impaction (right or left), root resorption in the lateral incisor and registration of adjacent teeth affected by resorption radicular (central incisor and/or premolars). In addition, when reabsorption of IL was observed, three-dimensional analysis was made using the Three-Dimensional Leeds Orthodontic Root Resorption Target Scale (3D-LORTS). The ICM in the selected sample had an average age of 12 years, a ratio by sex of women:men of $1.5: 1$, frequently of unilateral type, on the right side and with palatine buccolingual location $(64.1 \%)$ above the vestibular (35.9 \%). Root resorption reached $35.9 \%$, of which $27.6 \%$ presented in the IL and 8.3 $\%$ in adjacent teeth. Regarding the three-dimensional distribution of root resorption, the most frequent involved the apical third (60\%), with pulpal or severe damage (40\%), in only one location, the palatine (54\%). None of the above variables considered showed statistical differences with the root resorption of lateral incisors.

KEY WORDS: impacted canine, tooth, cone beam, root resorption.

\section{REFERENCIAS BIBLIOGRÁFICAS}

Alqerban, A.; Jacobs, R.; Lambrechts, P.; Loozen, G. \& Willems, G. Root resorption of the maxillary lateral incisor caused by impacted canine: a literature review. Clin. Oral Investig., 13(3):247-55, 2009.

Arriola-Guillén, L. E.; Ruíz-Mora, G. A.; Rodríguez-Cárdenas, Y. A.; Aliaga-Del Castillo, A. \& Dias-Da Silveira, H. L. Root resorption of maxillary incisors after traction of unilateral vs bilateral impacted canines with reinforced anchorage. Am. J. Orthod. Dentofacial Orthop., 154(5):645-56, 2018.

Becker, A. \& Chaushu, S. Etiology of maxillary canine impaction: a review. Am. J. Orthod. Dentofacial Orthop., 148(4):557-67, 2015.

Bizcar, M. B.; Sandoval, V. P. \& Navarro, C. P. Radiographic analysis and prevalence of impacted maxillary canine teeth in children between 8 and 16 years. Int. J. Odontostomat., 9(2):283-7, 2015. 
MUÑOZ, D. M.; ARRAYA, V. D.; CASTRO, C. D. \& VERGARA, N. C. Impactación canina maxilar y reabsorción radicular de dientes adyacentes: un análisis a través de tomografía computarizada cone-beam. Int. J. Odontostomat., 14(1):27-34, 2020.

Cernochova, P.; Krupa, P. \& Izakovicova-Holla, L. Root resorption associated with ectopically erupting maxillary permanent canines: a computed tomography study. Eur. J. Orthod., 33(5):483-91, 2011.

Dewel, B. F. The upper cuspid: Its development and impactation. Angle Orthod., 19(2):79-90, 1949.

Dogramaci, E. J.; Sherriff, M.; Rossi-Fedele, G. \& McDonald, F. Location and severity of root resorption related to impacted maxillary canines: a cone beam computed tomography (CBCT) evaluation. Aust. Orthod. J., 31(1):49-58, 2015.

Ericson, S. \& Kurol, J. Radiographic examination of ectopically erupting maxillary canines. Am. J. Orthod. Dentofacial Orthop., 91(6):48392, 1987.

Ericson, S. \& Kurol, P. J. Resorption of incisors after ectopic eruption of maxillary canines: a CT study. Angle Orthod., 70(6):415-23, 2000.

Herrera-Atoche, J. R.; Agüayo-de-Pau, M. D.; Escoffié-Ramírez, M.; Aguilar-Ayala, F. J.; Carrillo-Ávila, B. A. \& Rejón-Peraza, M. E. Impacted maxillary canine prevalence and its association with other dental anomalies in a Mexican population. Int. J. Dent., 2017:7326061, 2017.

Jacoby, $\mathrm{H}$. The etiology of maxillary canine impactions. Am. J. Orthod., 84(2):125-32, 1983.

Jawad, Z.; Carmichael, F.; Houghton, N. \& Bates, C. A review of cone beam computed tomography for the diagnosis of root resorption associated with impacted canines, introducing an innovative root resorption scale. Oral Surg. Oral Med. Oral Pathol. Oral Radiol., 122(6):765-71, 2016.

Lai, C. S.; Bornstein, M. M.; Mock, L.; Heuberger, B. M.; Dietrich, T. \& Katsaros, C. Impacted maxillary canines and root resorptions of neighbouring teeth: a radiographic analysis using cone-beam computed tomography. Eur. J. Orthod., 35(4):529-38, 2013.

Liu, D. G.; Zhang, W. L.; Zhang, Z. Y.; Wu, Y. T. \& Ma, X. C. Localization of impacted maxillary canines and observation of adjacent incisor resorption with cone-beam computed tomography. Oral Surg. Oral Med. Oral Pathol. Oral Radiol. Endod., 105(1):91-8, 2008.

Sacerdoti, R. \& Baccetti, T. Dentoskeletal features associated with unilateral or bilateral palatal displacement of maxillary canines. Angle Orthod., 74(6):725-32, 2004.

Stewart, J. A.; Heo, G.; Glover, K. E.; Williamson, P. C.; Lam, E. W. \& Major, P. W. Factors that relate to treatment duration for patients with palatally impacted maxillary canines. Am. J. Orthod. Dentofac. Orthop., 119(3):216-25, 2001.

Walker, L.; Enciso, R. \& Mah, J. Three-dimensional localization of maxillary canines with cone-beam computed tomography. Am. J. Orthod. Dentofac. Orthop., 128(4):418-23, 2005.

Yan, B.; Sun, Z.; Fields, H.; Wang, L. \& Luo, L. Etiologic factors for buccal and palatal maxillary canine impaction: a perspective based on cone-beam computed tomography analyses. Am. J. Orthod. Dentofac. Orthop., 143(4):527-34, 2013.
Dirección para correspondencia:

Daniel Arraya Valdés

Cirujano Dentista

Facultad de Odontología

Universidad Andrés Bello

Viña del Mar

CHILE

Email: d.arrayavaldes@gmail.com

Recibido : 08-06-2019

Aceptado: 19-08-2019 